REVIEW ARTICLE

\title{
Sensory processing disorders - diagnostic and therapeutic controversies
}

\author{
Aneta R. Borkowska ${ }^{E, F}$ \\ Maria Curie-Skłodowska University, Lublin, Poland
}

This article presents the current state of knowledge regarding the controversial issue of sensory integration dysfunction/sensory processing disorder. Symptoms are defined as impairments in the accurate reception and registering of stimuli, differentiation of stimulus intensity, and adequate reactivity to stimulation. They can be of specific character and occur in isolation and can also be a nonspecific element of a clinical picture of another disease entity. Psychophysiological and neuroimaging studies confirm the existence of both a distinct group of children with symptoms of sensory processing disorder diagnosed based on descriptions of behaviours listed in questionnaires and of a specific neurobiological basis of this disorder. In clinical practice, it is of key importance to determine whether behavioural problems observed in children are caused by disorders other than sensory processing disorders. Results of meta-analyses regarding sensory integration therapy are inconclusive and do not allow this form of treatment to be considered factbased. Future studies with high methodological standards are necessary in order to verify the effectiveness of different forms of sensory integration therapy. Parents should be informed about the existing limitations.

\section{KEY WORDS}

sensory processing; sensory integration; neuronal correlates; SI therapy

Corresponding Author - Aneta R. Borkowska, Maria Curie-Skłodowska University, 5 Maria Skłodowska-Curie Square, 20-001 Lublin, Poland, e-mail: aneta.borkowska@poczta.umcs.lublin.pl

AUthors' CONTRIBUtion - A: Study design - B: Data collection · C: Statistical analysis · D: Data interpretation .

E: Manuscript preparation · F: Literature search · G: Funds collection

TO CITE THIS ARTICLE - Borkowska, A. R. (2017). Sensory processing disorders - diagnostic and therapeutic controversies.

Current Issues in Personality Psychology, 5(3), 196-205.

RECEIVED 27.03.2017 · REVIEWED 03.07.2017 • ACCEPTED 08.07.2017 · PUBLISHED 22.09.2017 


\section{BACKGROUND}

Sensory integration disorder (SI) has been present in clinical practice for several decades (Adams, Feldman, Huffman, \& Loe, 2015; Ayres, 1989; Cheung \& Siu, 2009; Miller, Anzalone, Lane, Cermak, \& Osten, 2007). The past two decades have seen a significant increase in the popularity of both diagnosing sensory integration disorders and applying SI therapy. However, popularity in clinical practice is not equivalent to popularity in research, which results in a lack of high quality research and publications which could confirm the appropriateness and usefulness of diagnostic-therapeutic activity in this area. This paper aims to present the current state of knowledge with regards to several important issues: the presence of symptoms and diagnosis of sensory integration disorder, the neural bases of the disorder symptoms, comorbidity with other neurodevelopmental disorders, and the effectiveness of SI therapy.

\section{SYMPTOMATOLOGY OF SENSORY PROCESSING DISORDER}

The term 'sensory integration', a basic notion in the concept of sensory disorders, was introduced by the occupational therapist A. Jean Ayres (1920-1989) in 1963 (Miller, Anzalone, Lane, Cermak, \& Osten, 2007). It referred to the way in which an organism deals with processing information from its environment as a sensory input. Sensory systems, like other functional systems (e.g. language and motor systems), are sensitive to developmental changes and factors which modify them. Thus, they can be fully accurate at orienting a child in the outside world, or, as a result of interference during development, they may cause changes in the way stimuli are received or in the quality of the reaction to those stimuli.

A well-organized sensory system should integrate information coming from all of the modalities: sight, hearing, proprioception, and the vestibular system. According to the originator of this concept, A. Jean Ayres, SI dysfunction leads to broad developmental deficits, especially in learning and emotion regulation processes (Ayres, 1989).

Nowadays, the term sensory processing disorder (SPD), rather than SI, is increasingly common in the literature. Sensory processing is the organisation and interpretation of sensory stimuli coming from the body and the environment, and symptoms of atypical sensory processing manifest themselves in inappropriate responses to sensory stimulation. Disorders of an SPD character are defined as a lack of ability to use information received by the senses in order to efficiently function in everyday life (Adams et al., 2015).
Modifying the approach of Ayres, Miller et al. (2007) proposed a classification of such disorders. This taxonomy indicates the need for distinguishing subtypes of dysfunctions of sensory processing, and also points out the existence of individual differences with regards to detection, regulation, interpretation, and reaction to experience/sensory input. They proposed that a diagnosis of SPD should only be made when difficulties in sensory processing are a significant hindrance in everyday life (Miller et al. 2007). Figure 1 presents the classification of SPD subtypes.

They are categorized in the following way: Sensory modulation disorder (SMD) subtype 1 - sensory over-responsivity (SOR) manifests itself as faster and longer-than-usual response to sensory stimuli. It may be manifested in either one (e.g. only touch) or several sensory systems. This way of reacting is especially visible in new, sudden, or unexpected situations. It also depends on many variable individual and contextual factors. It is an unconscious, physiological reaction to stimuli.

SMD subtype 2 - sensory under-responsivity (SUR) is characterised by lack of reaction to sensory stimuli. Affected children seem not to perceive the stimuli they are exposed to; they do not even react to pain or significantly lowered temperatures. As a result of this, children seem to lack interest in the outside world - living in their own world, isolated and lacking motivation for activity. Under-responsivity is rarely diagnosed in small children, because they seemingly do not exhibit behavioural problems and are 'good' and 'well-behaved'.

SMD subtype 3 - sensory seeking (SS) manifests as atypical, excessive seeking of distinct, strong stimulation in various modalities, e.g. preference for strongly spiced food, making a lot of noise during play, or preference for the presence of many visual stimuli in one's surroundings. Such children's behaviour is assessed by people in their social environment as risky, naughty and impulsive. In order to provide themselves with a sufficient amount of stimulation, children may exhibit repetitive behaviours (i.e. perseverations).

Sensory discrimination disorder (SDD). Individuals with this type of sensory processing disorder exhibit difficulties with qualitative interpretation of sensory stimuli and are not able to recognize similarities and differences between stimuli. The fact that a stimulus occurs is registered and regulation of the response to the stimulus is also possible. However, precise recognition of the quality of a stimulus is a problem. Difficulties of this type may concern one or many sensory systems.

Sensory-based motor disorder (SBMD) subtype 1 - postural disorder is characterised by difficulties in stabilization of the body during movement or at rest. Inadequate muscle tension, hypotonia or hypertonia, inaccurate movement control, poor body stability, 


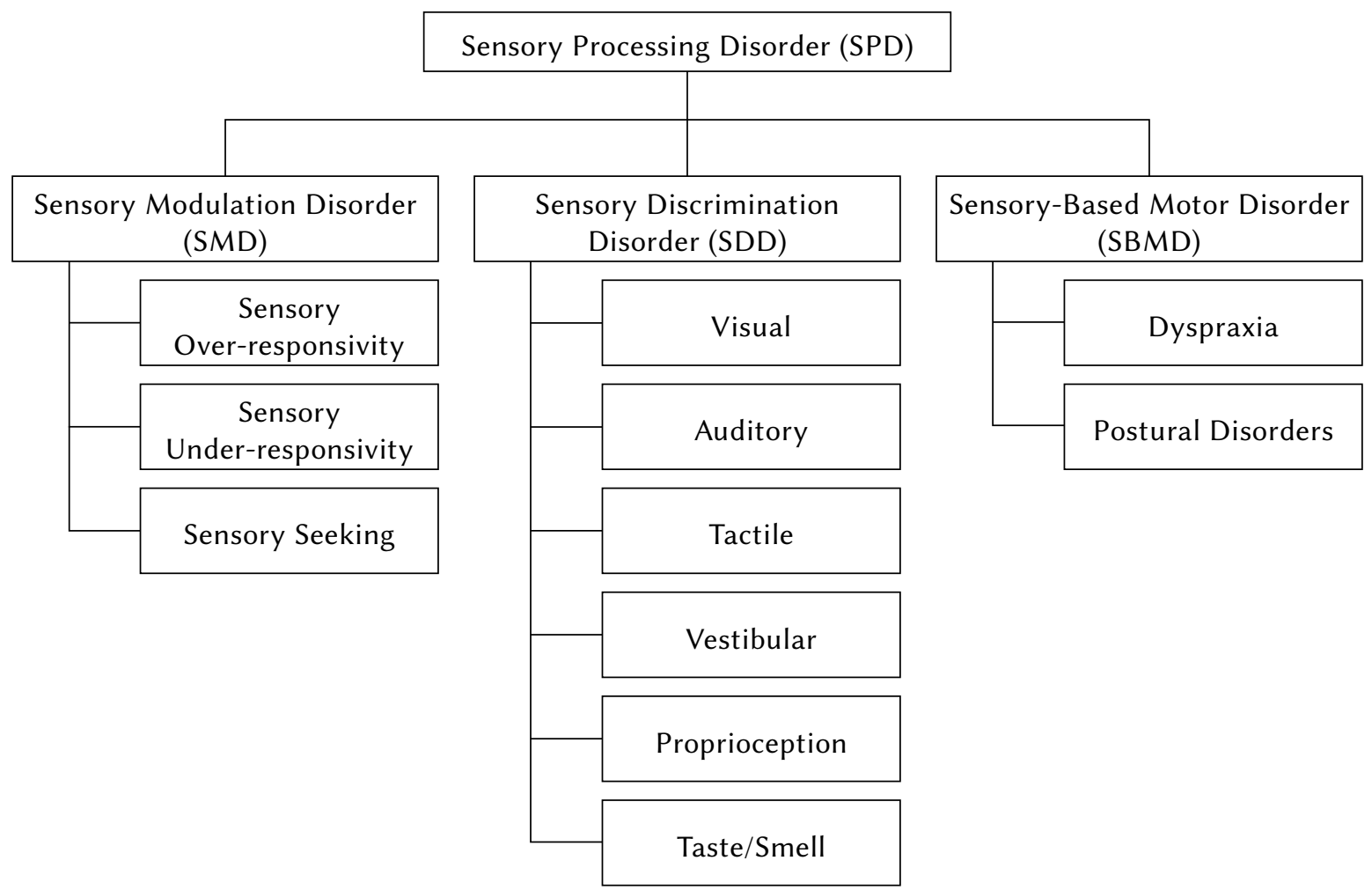

Figure 1. Subtypes of sensory processing disorders, according to Miller et al. (2007).

difficulties with moving one's weight from one leg to the other, etc., are observed.

SBMD subtype 2 - dyspraxia is manifested in impairments in planning motor sequences and performing new motor activities. Such individuals have difficulties with coordination, precision, and harmony of movements with regards to gross and fine motor skills, as well as oral praxis (Miller et al. 2007).

Given the above descriptions of symptoms which define SPD, one could observe that, at the level of analysing the observed behaviours, symptoms are analogous or similar to those characteristic of disorders such as attention deficit hyperactivity disorder (ADHD), autism spectrum disorders, or developmental coordination disorder (DCD).

\section{SENSORY PROCESSING DISORDER AS A SET OF SYMPTOMS OR A NOSOLOGIC ENTITY}

There is a large body of studies which confirm the occurrence of symptoms interpreted as behaviours caused by sensory processing disorders, diagnosed on the basis of observational questionnaires completed by caregivers as well as evaluations using the Southern California Tests (diagnostic trials assessing various aspects of sensory processing) (Cheung \& Siu, 2009; Tomchek \& Dunn, 2007). Despite this, SPD does not exist as a single nosological entity in international classifications such as DSM-5 or
ICD-10. However it is included as a regulatory-sensory processing disorder in:

1. Zero To Three (2005). Diagnostic Classification of Mental Health and Developmental Disorders of Infancy and Early Childhood: Revised Edition (dc:0-3r).

2. Diagnostic Manual for Infancy and Early Childhood (2005). Mental Health, Developmental, Regulatory-Sensory Processing and Language Disorders and Learning Challenges.

Thus, there are no formal reasons to diagnose SPD in children over 3 years old.

\section{NEUROPHYSIOLOGICAL INDICATORS OF SENSORY PROCESSING DISORDER}

The results of neuropsychological and neuroimaging studies on a group of children selected based on behavioural indicators (i.e. the symptoms described in symptom scales) are an objective indicator of the presence of problems with sensory processing. One such neurophysiological indicator is the level of sensory gating, which is a natural brain reaction suppressing signals which are redundant or unimportant and selectively directing one's own sensitivity to sensory stimuli (Davies \& Gavin, 2007). Thus it is a basic psychophysiological mechanism of brain functioning, directing one's processing resources towards important environmental stimuli (Myles-Worsley et al., 1996). The P50 and N100 indices, measured by 
recording the bioelectrical activity of the brain when examining event-related potentials (ERPs) associated with hearing, are used as a measurement of sensory gating. The P50 and N100 indices are reactions to ultra short $3 \mathrm{~ms}$ auditory stimuli presented at intervals of $500 \mathrm{~ms}$. In a study examining ERP measurements in 25 typically developing children and 28 children diagnosed with SPD, on the basis of the general score on the Short Sensory Profile, aged 5-12, children with SPD exhibited weaker gating and more variability of reaction in comparison to typically developing children. This suggests that children with SPD exhibit deficits in filtering repetitive auditory stimuli and they cannot selectively regulate their sensitivity to sensory stimuli.

Developmental changes in the gating function, which increased with age in the control group, were also observed. This was not observed in the SPD group. Brain activity correctly differentiated children with SPD from normally developing children with $86 \%$ precision. These results are evidence that children with SPD have different mechanisms of reception of sensory information to their peers, which may explain the symptoms of the disorder and in this way confirm the value of the SPD diagnosis (Davies, Chang, \& Gavin, 2009; Davies \& Gavin, 2007).

Neurophysiological studies using ERP also indicate that functional impairments in both the cortical areas engaged in the processing of sensory information and dysfunctions of higher-order processes important for multimodal sensory integration (MSI) may lie at the base of SPD (Brett-Green, Miller, Schoen, \& Nielsen, 2010).

Research by Owen et al. (2013) proved to be very interesting and informative for understanding the neural basis of SPD. They analysed the specifics of the microstructure of white matter of children with SPD in studies using the diffusion tensor imaging (DTI) technique. Characteristics of the microstructure of the tracks and pathways which constitute white matter, such as the axonal diameter, fibre density, and myelination, determine the speed and the bandwidth of information transmission in the human brain (Mukherjee, Berman, Chung, Hess, \& Henry, 2008). Working under the assumption that structural impairments in the primary sensory cortex fibres or fibres connecting the multimodal association areas may result in a loss of precision in temporal spread of activation, which is crucial for the precise processing of the unimodal and multimodal sensory input, Owen et al. (2013) tried to assess the structural features of white matter in children with SPD. The following indices were used to assess the microstructure: a) fractional anisotropy (FA), which indicates the variability of directions of water diffusion in white matter, in order to obtain data reflecting the microstructure properties, i.e. axonal diameter, myelination levels, and fibre density; b) mean diffusivity
(MD), i.e. the speed of diffusion averaged over all directions; and c) radial diffusivity $(\mathrm{RD})$, i.e. the speed of diffusion perpendicular to the orientation of fibres. The normal maturation of white matter in childhood involves an increase in FA and a decrease in MD and RD (Yoshida, Oishi, Faria, \& Mori, 2013). Significant differences were observed in all measures of reduced integration of the microstructure of white matter (i.e. in FA, MD and RD). A decrease in FA was observed in the SPD group in the corpus callosum, left posterior projections of the thalamus, the corona radiata, and posterior parts of the left dorsal longitudinal fasciculus. Additionally, the area of right projections of the thalamus and corona radiata exhibited a strong tendency towards lowered FA in the SPD group. In the case of the MD and RD indices, an increase in their value was observed in children with SPD in the lateral fibres of the corpus callosum, in the splenium, bilaterally in thalamus projections, optic radiation, right corona radiata, and in the fibres of the longitudinal fasciculus. The existence of significant correlations between the specifics of the indicated areas of white matter and behavioural indices was also revealed. No differences were found in terms of volumetry between SPD and TD children with regards to white and grey matter, which suggests that the pathology does not concern the macrostructure (Owen et al., 2013).

Thus, psychophysiological and neuroimaging studies confirm the existence of a distinct group of children with a sensory processing disorder which had previously been thought of as a behavioural disorder. They also suggest the specificity of the neurobiological basis of this disorder.

\section{SENSORY PROCESSING DISORDER AND OTHER NEURODEVELOPMENTAL DISORDERS}

Taking into account the fact that similar symptoms may have a different etiology and varying mechanisms, it is controversial whether the symptoms of SPD constitute an isolated set of symptoms or whether they are an element of the clinical picture of another disorder. This issue has been tackled in research which compared children with SPD symptoms and no other diagnoses, children with other disorders, and typically developing children.

Many studies indicate that symptoms characteristic of sensory processing disorders (observed in the behaviour of a child) are much more frequently diagnosed within existing diagnostic criteria than in the general population. This is because in the general population the occurrence of SPF is assessed at $5-16 \%$, while in clinical samples, e.g. of autism spectrum disorders (ASD), the symptoms are observed in as many as $90 \%$ of children (Owen et al., 2013). 
The highest number of studies analysing the symptoms of sensory disorders were devoted to children with autism. This is likely because this problem frequently occurs in ASD, which was reflected in the DSM-5, where it was included in the diagnostic criteria (APA, 2013).

In research dedicated to the assessment of levels of sensory processing in autistic children (Takarae, Sablich, White, \& Sweeney, 2016), internal variability within the group was shown in terms of the levels of sensory oversensitivity. Psychophysiological methods were applied, consisting of the assessment of visual response to the manipulation of contrast. The resulting increased neuronal reactivity was interpreted as the cause of sensory oversensitivity in ASD. A higher value of visual response than in the control group was observed in most, but not all, children with ASD. The authors suggest that individuals in whom a pattern of higher neural activity is observed may constitute a distinct subgroup of ASD, requiring a modified therapeutic approach.

The first psychophysiological study directly comparing children with autism, children with sensory modulation disorders (SMD, a subtype of SPD), and typically developing children, was published by Schoen et al. in 2009. Both clinical groups were compared with regards to skin conductivity levels registered before applying the stimuli (tonic arousal measurement) and during stimulation (phasic reactivity measurement). The Short Sensory Profile questionnaire, completed by parents, was used to assess sensorially determined behaviours. In terms of the indices of tonic arousal, children with ASD were significantly different from those with SMD and typically developing children, but those with SMD did not differ from the typically developing children. However, reactivity, especially registered after the first stimulus, was higher in the SMD group, independent of the type of stimulus. Reactivity to stimuli in the ASD group was the lowest in all groups. Thus, children with ASD exhibited atypical arousal and children with SMD exhibited atypical reactivity to stimuli. In the Short Sensory Profile symptoms questionnaire, both clinical groups exhibited significantly more sensorially determined dysfunctional behaviours than did the typically developing children. The ASD group had a significantly higher overall index of sensory impairments than did the SMD group. The profiles of symptoms looked different. The ASD group had higher indices on the subscale of sensitivity to taste and smell, while the SMD group scored higher on the sensory seeking subscales. These results are a basis for treating these two disorders as separate (Schoen, Miller, Brett-Green, \& Nielsen, 2009).

Similarity in terms of sensory processing problems was observed between groups with autism and Tourette syndrome, as reported by Ludlow and Wilkins (2016). Their paper found that both groups had atyp- ical abilities of sensory modulation and atypical abilities to increase or suppress responses to sensory stimuli. They concluded that these symptoms were a common feature of subjects from both clinical groups and may constitute the mechanism common to the two disorders. Additionally, sensory impairments, mainly tactile, auditory and visual over-responsiveness, were diagnosed in children with Tourette syndrome, which had a significant impact on the manifestation of tic-type symptoms. The authors suggested that Tourette syndrome is a result of cortical over-excitability. This is in line with previously published research, in which it was reported that tics are preceded by some form of stimulation. The type and intensity of stimuli which influence the onset of tics may be very individual.

Children with ADHD are another clinical group which has been analysed with regards to impairments in sensory processing. Miller et al. (2012) compared children diagnosed with isolated ADHD, children diagnosed with isolated SMD, and children with a double diagnosis. The results suggested that all clinical groups have greater difficulties with the functioning of attention, higher indices of impulsivity, sensory difficulties, and difficulties with activity and motor skills, but each group had a different profile of these impairments. The attention deficit indices in the ADHD group were higher in comparison to the SMD group. Children with a double diagnosis exhibited more behaviours caused by sensory impairments than those with ADHD and more attentional difficulties than children with SMD. On this basis, the authors concluded that ADHD and SMD are separate disorders.

Similar conclusions were formulated on the basis of a longitudinal study conducted in order to determine the developmental pathways of hyperactivity symptoms and symptoms of over-responsivity to stimuli. Ben-Sasson, Soto, Heberle, Carter, and Briggs-Gowan (2014) found that the symptoms of sensory over-responsivity (SOR) and ADHD appear independently and are stable over time. The research was done with an initial group of 922 infants. The children were assessed three times in the period between infancy and early school age with regards to social functioning, emotional functioning, sensory sensitivity, attention and impulsivity/hyperactivity. When the children reached school age, they formed four clusters: those with increased sensory over-responsivity symptoms (SOR), those with increased ADHD symptoms (ADHD), those with increased SOR and ADHD symptoms (ADHD+SOR), and those with low values of ADHD and SOR symptom indices. Children from the SOR and from the ADHD+SOR group were characterised by higher indices of sensory sensitivity in early childhood in comparison to children from the ADHD group and those without symptoms. The ADHD and ADHD+SOR groups 
differed from the SOR group and the asymptomatic group in terms of increased symptoms of impulsivity/hyperactivity as well as inattention. The authors concluded that symptoms of SOR and ADHD appear independently and are stable over time. This conclusion is in line with the results of Lane, Reynolds, and Thacker (2010), who, on the basis of an assessment of cortisol levels, skin conductivity, and behavioural indices, concluded that the symptoms of these disorders are separate problems. The research also found the highest levels of anxiety in the group with comorbid symptoms of ADHD and SOR, which suggests an accumulation of problems in life of individuals affected by the two disorders.

In a study whose goal was to compare ADHD and autism with comorbid behaviours from the SPD spectrum (Sanz-Cervera, Pastor-Cerezuela, Fernández-Andrés, \& Tárraga-Mínguez, 2015), it was found that the features of SPD were significant predictors of the severity of autism, and they exerted a similar influence on the development of behaviours at home and in the school environment. In ADHD, SPD features had a greater influence on behaviour at home than at school. They also predicted both inattention and hyperactive/impulsive behaviours. Thus, symptoms of SPD specifically modify the clinical picture of the disorders with which they occur.

In a study by Cheung and Siu (2009), two clinical groups, children with ASD and with ADHD, were compared to children with no disorders. Behaviours suggesting problems with sensory processing were analysed using the Chinese Sensory Profile (CSP). Children with developmental disorders scored significantly higher on indices suggesting greater problems in sensory processing than healthy children. It was also found that while the CSP is good at differentiating between healthy children and children with disorders, it does not differentiate between the two clinical groups. No gender differences were found, but age was found to be a significant variable. A relatively small decrease in the intensity of symptoms was observed in children with ASD between the ages of 6 and 12. A similar tendency was observed in healthy children. However, an increase in the intensity of symptoms was observed in children with ADHD. The authors concluded that sensory processing disorders may be a nonspecific indicator of developmental dysfunctions in children because they significantly differentiate between children with disorders and healthy children, but that they do not differentiate between the clinical groups.

The presented research suggests that behaviours evincing difficulties in sensory processing may constitute nonspecific indicators of developmental difficulties. This hypothesis is in line with the lack of differences in this type of behaviour between children with ADHD, ASD, and Tourette's. At the same time it turns out that children with sensory processing disor- ders may constitute a distinct group showing symptoms of only that disorder. Symptoms of SPD may also co-occur with diagnoses such as ASD or ADHD. Due to this, a differential diagnosis is of particular importance. However, the results published to date are not sufficient to determine specific characteristic profiles of behaviour disorders, which could be some kind of marker of a given developmental problem and be a basis for differentiation. Taking this fact into account, it should be stressed that symptoms of disturbed, dysfunctional behaviour incorrectly interpreted as SPD may lead to incorrect diagnoses and result in wrong directions of treatment. For instance, if a child presents intense involuntary movements and perseverations, and these are considered to stem from a sensory processing disorder related need for stimulation, but in reality are the result of inflammatory changes in the central nervous system, the consequences of such a misdiagnosis may be very serious. Thus, the first step in the diagnosis of a child with symptoms of impeded behaviour is to search for the possibility of diagnosing a disorder that exists in the international classifications of diseases (Zimmer et al., 2012).

\section{EFFECTIVENESS OF THERAPY}

The idea of evidence-based practice suggests that only fact-based treatments should be used on patients (Cierpiałkowska, 2016). This means that it is necessary to take into account not only clinical experience, but also scientific research. Thus, studies concerned with the success of SI therapy ought to be a basis for using such kinds of treatment on children. Studies evaluating the effects of treatment are concerned with either efficacy or effectiveness. The first is done in a laboratory setting and its goal is to determine the strength of influence of tailored interventions on the impaired aspect of behaviour. Effectiveness trials are done in real-world conditions, without the possibility to control for many independent variables. They assess the influence of only roughly characterised independent variables (therapy in real-world conditions is always tailored to the individual and cannot be homogenised) on dependent variables, which are also often inconsistently operationalised. In comparison to efficacy studies, effectiveness studies have significantly higher external validity, but lower internal validity (Cierpiałkowska, 2016). None of the studies published on SI are efficacy trials (they are all effectiveness trials). An assessment of the usefulness of SI therapy is possible only on the basis of meta-analyses, which provide data allowing one to make valid inferences. Analysis of the results of meta-analyses is especially important, due to the great popularity, even commonplaceness, of this form of therapy.

The first such meta-analysis (Vargas \& Camilli, 1999) showed that the SI effect size in comparison to 
no therapy, assessed in publications dated 1983-1993, was very low (0.03) and not statistically significant. Shaw, Powers, Abelkop, and Mullis (2002) published the results of a meta-analysis of 41 studies, including 218 effect sizes. There were no significant effects for improvement of language $(-0.08)$, indices of behaviour $(0.02)$, or sensorimotor functions $(-0.10)$. Small but significant effects were found for motor functions (0.24) and school performance (0.26). However, when the factor of development was taken into account, the effect in both areas dropped to 0.03 and -0.04 respectively. Another meta-analysis, by Schaaf and Miller (2005), included over 80 studies devoted to measuring various aspects of the success of sensory integration intervention in developmental disorders. Only about half of these studies confirmed some type of treatment effectiveness. Parham et al. (2007) noted a lack of precision in studies on SI therapy. Their meta-analysis investigated the treatment process indices used in studies. They identified 10 elements of therapy and then analysed 34 studies with regards to their presence in the descriptions of therapy. In most studies, the elements of the therapy's structure were described, though of these 10 elements, only one was present in all of the studies. Most of these mentioned fewer than half of these elements. Descriptions of interventions in $35 \%$ of the studies were inconsistent with some element of the process. Parham et al. stressed that the descriptions of the therapy process were insufficient, and until this condition is met, inferences regarding the effectiveness should not be considered as trustworthy.

Sensory interventions are often used with children with autism, and so some of the meta-analyses refer only to studies devoted to measuring the effectiveness of this treatment only in this group of children (Devlin, Healy, Leader, \& Hughes, 2011; Schaaf et al., 2014; Sniezyk \& Zane, 2015). The conclusions of these meta-analyses mention the problems with the selection of studies to meta-analyse, as many studies are not sufficiently scientifically rigorous. Thus, despite the fact that the procedures of sensory therapy are used all over the world, there is still no literature which can present methodologically sound studies of its effectiveness in autism. Secondly, the results of those studies which were rigorous revealed that there is no causal relationship between sensory therapy and improvement in the assessed variables (i.e. different dimensions of behaviour of children with autism). In studies comparing behavioural therapy and SI therapy, some significant improvement in the difficult symptoms of autism was observed, but greater effects were observed with regards to behavioural therapy. When only behavioural intervention was used, there was visible improvement of difficult symptoms, including self-harm. The authors concluded by confirming that sensory procedures cannot be considered to be based in fact. The research to date is at best inconclusive, and at worst shows how far this therapy still is from being effective (Sniezyk \& Zane, 2015). Thus, the use of SI therapy to treat autism remains unsubstantiated.

In recent years, studies assessing and comparing the effectiveness of two treatment approaches, Ayres' Sensory Integration (ASI) and sensory-based interventions (SBIs), both within the sensory integration therapy framework, have emerged (Watling \& Hauer, 2015; Yunus, Liu, Bissett, \& Penkala, 2015). ASI is a system, developed by Ayres and further expanded by her students and successors, based on broad treatment in order to improve the integration of sensory systems and thereby improve the child's functioning. SBIs are interventions tailored to deal with specific sensory systems. The two treatment systems were compared by Watling and Hauer (2015). A meta-analysis of 23 articles published between 2006 and 2013 showed that there is a moderate effect for ASI; however, the results for SBIs were inconclusive. The authors recommend conducting future research on ASI and SBIs with higher care for methodological correctness, bigger sample sizes, and better described measures.

A meta-analysis of studies on the effectiveness of SBIs was published by Yunus et al. (2015). Of 132 papers identified as dedicated to this issue, only 14 met the criteria for inclusion in the analysis. Seven of these were devoted to tactile interventions, 4 to proprioceptive interventions, and 3 to vestibular interventions. Tactile interventions such as massages turned out to be the most promising for reduction of problematic behaviours in children. However the general conclusion from the studies does not allow for a sense of certainty with regards to evidence for the effectiveness of SBIs. In both papers (Watling \& Hauer, 2015; Yunus et al., 2015) suggestions are made to improve the quality of the research on SI intervention. The proponents and users of SI therapy, being aware of the serious necessity to introduce objective measures of its effects, are already making attempts to implement these suggestions. Among other things, a psychometric tool was developed to evaluate the effectiveness of the ASI intervention: the Fidelity Measure for Research on the Effectiveness of the Ayres Sensory Integration Intervention (Parham et al., 2011).

In a monograph devoted to controversial therapies in developmental disorders, Smith, Mruzek, and Mozingo (2016) hypothesise that the reason for problems with effectiveness trials is an underdeveloped theoretical basis, leading to unvalidated means of assessment. For instance, speculative theoretical constructs are used, such as the concept of vestibular disorder. The authors also pointed to the possible negative outcomes of using SI therapy, mentioning an experiment in which a 4-year-old autistic child exhibiting intense outbursts of anger took part. During 
the breaks in educational tasks, during the control procedure, the boy played with his favourite toys, and during the experimental procedure his mother brushed her son, as recommended by the SI therapist. The person describing the boy's symptoms of anger did not know the information about the phase of the trial. Intensification of undesirable symptoms as a result of the SI therapy exercises was observed. This result contradicts the assumption of no risk and no possibility of causing harm as an effect of using this therapy. The early papers by Ayres state that this is a natural therapy based on a holistic approach to the child, and that there are no risks associated with its use. However, such a claim ignores the fact that ineffective treatment takes time and diverts attention and focus from forms of therapy that could be more effective (Smith, Mruzek, \& Mozingo, 2016).

In 2012, the American Academy of Pediatrics (AAP) published a Policy Statement in which they expressed concern about the increasing numbers of diagnoses of sensory processing disorder and the use of sensory processing therapies by occupational therapists and other specialists working with children affected by developmental disorders (Zimmer et al., 2012). The AAP very clearly recommends not diagnosing SPD and instead searching for other explanations, in line with existing nosologic entities, for behavioural problems in children. With regards to therapy, the AAP indicates that it can be accepted as one component of a therapy plan and treatment of a child. However, parents should be informed in each instance that the number of studies on the success of SI therapy is limited, and the results are inconclusive. They should also have the chance to try out such interventions and check whether they are of benefit to the child. Publication of this paper led to serious discussion and many comments, including from researchers who agree with the statement presented therein (Comments section of "Sensory Integration Therapies for Children With Developmental and Behavioral Disorders”, 2012).

\section{CONCLUSIONS}

This paper has discussed the controversial issue of sensory processing disorders in the context of the specifics of symptoms, their psychophysiological and neural correlates, differential diagnosis, as well as therapeutic interventions. At the moment it is not possible to make a formal diagnosis of SPD because these disorders do not exist as diagnostic entities either in DSM-5 or ICD-10. The literature review suggests that symptoms of such disorders (defined as impairments in the accurate registration of stimuli, correct reception and differentiation of the intensity of stimuli, and adequate reactivity to stimulation) may be of a specific character and occur in isolation, but that they may also be a nonspecific element of the clinical picture of other disease entities. In clinical practice, it is of key importance to discern whether behavioural problems observed in children cannot be explained by causes other than sensory processing disorders, and this need stems from the ambiguity of symptoms in neurodevelopmental disorders (Chrzan-Dętkoś, Pawlicka, \& Bogdanowicz, 2014; Grzegorzewska, Pisula, \& Borkowska, 2016; Pawlicka, Lipowska, \& Gajdzińska, 2015; Trempała \& Cieciuch, 2016). Meta-analyses concerned with SI therapy published to date are inconclusive and do not allow for this form of intervention to be considered fact-based. Further research, with the highest methodological standards, is vital to verify the successfulness of various forms of SI therapy. Parents choosing to use this therapy should be informed about the existing limitations.

\section{References}

Adams, J. N., Feldman, H. M., Huffman, L. C., \& Loe, I. M. (2015). Sensory processing in preterm preschoolers and its association with executive function. Early Human Development, 91, 227-233.

American Psychiatric Association. (2013). Diagnostic and statistical manual of mental disorders (DSM-5®). American Psychiatric Pub.

Ayres, A. J. (1989). Sensory integration and praxis tests (SIPT). Los Angeles: Western Psychological Services.

Ben-Sasson, A., Soto, T. W., Heberle, A. E., Carter, A. S., \& Briggs-Gowan, M. J. (2014). Early and concurrent features of ADHD and sensory over-responsivity symptom clusters. Journal of Attention Disorders, 21, 835-845.

Brett-Green, B. A., Miller, L. J., Gavin, W. J., \& Davies, P. L. (2008). Multisensory integration in children: A preliminary ERP study. Brain Research, 1242, 283-290.

Brett-Green, B. A., Miller, L. J., Schoen, S. A., \& Nielsen, D. M. (2010). An exploratory event-related potential study of multisensory integration in sensory over-responsive children. Brain Research, 1321, 67-77.

Cheung, P. P., \& Siu, A. M. (2009). A comparison of patterns of sensory processing in children with and without developmental disabilities. Research in Developmental Disabilities, 30, 1468-1480.

Comments section of "Sensory Integration Therapies for Children with Developmental and Behavioral Disorders". (2012). American Academy of Pediatrics. Retrieved from http://pediatrics.aappublications.org/content/129/6/1186.comments

Chrzan-Dętkoś, M., Pawlicka, P., \& Bogdanowicz, M. (2014). The effects of kangaroo mother care in a sample of preterm, preschool aged children. Health Psychology Report, 2, 208-217. 
Cierpiałkowska, L. (2016). Efektywność poradnictwa psychologicznego i psychoterapii [Effectiveness of psychological counseling and psychotherapy]. In L. Cierpiałkowska \& H. Sęk (eds.), Psychologia kliniczna [Clinical psychology] (pp. 727-738). Warszawa: Wydawnictwo Naukowe PWN.

Davies, P. L., \& Gavin, W. J. (2007). Validating the diagnosis of sensory processing disorders using EEG technology. The American Journal of Occupational Therapy, 61, 176-189.

Davies, P. L., Chang, W. P., \& Gavin, W. J. (2009). Maturation of sensory gating performance in children with and without sensory processing disorders. International Journal of Psychophysiology, 72, 187-197.

Devlin, S., Healy, O., Leader, G., \& Hughes, B. M. (2011). Comparison of behavioral intervention and sensory-integration therapy in the treatment of challenging behavior. Journal of Autism and Developmental Disorders, 41, 1303-1320.

Diagnostic Manual for Infancy and Early Childhood (2005). Mental Health, Developmental, RegulatorySensory Processing and Language Disorders and Learning Challenges (icdl-dmic). Bethesda, MD, Interdisciplinary Council on Developmental and Learning Disorders (ICDL).

Grzegorzewska, I., Pisula, E., \& Borkowska, A.R. (2016). Psychologia kliniczna dzieci i młodzieży [Child and adolescent clinical psychology]. In L. Cierpiałkowska \& H. Sęk (eds.), Psychologia kliniczna [Clinical psychology] (pp. 451-498). Warszawa: Wydawnictwo Naukowe PWN.

Lane, S. J., Reynolds, S., \& Thacker, L. (2010). Sensory over-responsivity and ADHD: differentiating using electrodermal responses, cortisol, and anxiety. Frontiers in Integrative Neuroscience, 4, 8, 1-11.

Ludlow, A. K., \& Wilkins, A. J. (2016). Atypical Sensory behaviours in children with Toutrette's Syndrome and in children with Autism Spectrum Disorders. Research in Developmental Disabilities, 56, 108-111.

Miller, L. J., Anzalone, M. E., Lane, S. J., Cermak, S. A., \& Osten, E. T. (2007). Concept evolution in sensory integration: A proposed nosology for diagnosis. American Journal of Occupational Therapy, 61, 135-140.

Miller, L. J., Nielsen, D. M., \& Schoen, S. A. (2012). Attention deficit hyperactivity disorder and sensory modulation disorder: A comparison of behavior and physiology. Research in Developmental Disabilities, 33, 804-818.

Mukherjee, P., Berman, J. I., Chung, S. W., Hess, C. P., \& Henry, R. G. (2008). Diffusion tensor MR imaging and fiber tractography: theoretic underpinnings. AJNR American Journal of Neuroradiology, 29, 632-641.

Myles-Worsley, M., Coon, H., Byerley, W., Waldo, M., Young, D., \& Freedman, R. (1996). Developmental and genetic influences on the P50 sensory gating phenotype. Biological Psychiatry, 39, 289-295.

Owen, J. P., Marco, E. J., Desai, S., Fourie, E., Harris, J., Hill, S. S., ...Mukherjee, P. (2013). Abnormal white matter microstructure in children with sensory processing disorders. Neuroimage: clinical, 2, 844-853.

Parham, L. D., Cohn, E. S., Spitzer, S., Koomar, J. A., Miller, L. J., Burke, J. P., ...Summers, C. A. (2007). Fidelity in sensory integration intervention research. The American Journal of Occupational Therapy, 61, 216-227.

Parham, L. D., Roley, S. S., May-Benson, T. A., Koomar, J., Brett-Green, B., Burke, J. P., ...Schaaf, R. C. (2011). Development of a fidelity measure for research on the effectiveness of the Ayres Sensory Integration ${ }^{\circledR}$ intervention. American Journal of Occupational Therapy, 65, 133-142.

Pawlicka, P., Lipowska, M., \& Gajdzińska, H. (2015). Linguistic stimulation impact on verbal working memory in the early stages of school education. Acta Neuropsychologica, 14, 49-60.

Sanz-Cervera, P., Pastor-Cerezuela, G., FernándezAndrés, M. I., \& Tárraga-Mínguez, R. (2015). Sensory processing in children with Autism Spectrum Disorder: relationship with non-verbal IQ, autism severity and Attention Deficit/Hyperactivity Disorder symptomatology. Research in Developmental Disabilities, 45, 188-201.

Schaaf, R. C., \& Miller, L. J. (2005). Occupational therapy using a sensory integrative approach for children with developmental disabilities. Developmental Disabilities Research Reviews, 11, 143-148.

Schaaf, R. C., Benevides, T., Mailloux, Z., Faller, P., Hunt, J., van Hooydonk, E., ...Kelly, D. (2014). An intervention for sensory difficulties in children with autism: A randomized trial. Journal of Autism and Developmental Disorders, 44, 1493-1506.

Schoen, S. A., Miller, L. J., Brett-Green, B. A., \& Nielsen, D. M. (2009). Physiological and behavioral differences in sensory processing: A comparison of children with autism spectrum disorder and sensory modulation disorder. Frontiers in Integrative Neuroscience, 3, 29, 1-11.

Shaw, S. R., Powers, N. R., Abelkop, S., \& Mullis, J. (2002). Sensory integration therapy: Panacea, placebo, or poison? Paper presented to the annual convention of the National Association of School Psychologists. Chicago, IL.

Smith, T., Mruzek, D. W., \& Mozingo, D. (2016). Sensory Integration Therapy. In J. A. Mulick \& R. M. Foxx (eds.), Controversial therapies for developmental disabilities: Fads, fasion and science in professional practice (pp. 247-269). Mahvah NJ: Lawrence Erlbaum.

Sniezyk, C. J., \& Zane, T. L. (2015). Investigating the effects of sensory integration therapy in decreasing stereotypy. Focus on Autism and Other Developmental Disabilities, 30, 13-22. 
Takarae, Y., Sablich, S. R., White, S. P., \& Sweeney, J. A. (2016). Neurophysiological hyperresponsivity to sensory input in autism spectrum disorders. Journal of Neurodevelopmental Disorders, 8, 29. doi: 10.1186/s11689-016-9162-9

Tomchek, S. D., \& Dunn, W. (2007). Sensory processing in children with and without autism: a comparative study using the short sensory profile. American Journal of Occupational Therapy, 61, 190-200.

Trempała, J., \& Cieciuch, J. (2016). The analysis of change in behavior and development: on some errors and possibilities to correct them. Current Issues in Personality Psychology, 4, 65-74.

Vargas, S., \& Camilli, G. (1999). A meta-analysis of research on sensory integration treatment. American Journal of Occupational Therapy, 53, 189-198.

Watling, R., \& Hauer, S. (2015). Effectiveness of Ayres Sensory Integration ${ }^{\circledR}$ and sensory-based interventions for people with autism spectrum disorder: A systematic review. American Journal of Occupational Therapy, 69, 6905180030p1-6905180030p12.

Yoshida, S., Oishi, K., Faria, A. V., \& Mori, S. (2013). Diffusion tensor imaging of normal brain development. Pediatric Radiology, 43, 15-27.

Yunus, F. W., Liu, K. P., Bissett, M., \& Penkala, S. (2015). Sensory-based intervention for children with behavioral problems: a systematic review. Journal of Autism and Developmental Disorders, 45, 3565-3579.

Zero To Three. (2005). Diagnostic Classification of Mental Health and Developmental Disorders of Infancy and Early Childhood: Revised Edition (dc:03r). Washington, DC, ZERO TO THREE Press.

Zimmer, M., Desch, L., Rosen, L. D., Bailey, M. L., Becker, D., Culbert, T. P., ...Adams, R. C. (2012). Sensory integration therapies for children with developmental and behavioral disorders. Pediatrics, 129, 1186-1189. 